\title{
Impulsive Differential Equations and Applications
}

\section{Katya Dishlieva*}

Faculty of Applied Mathematics and Informatics, Technical University of Sofia, Bulgaria

\section{Why We Study Impulsive Differential Equations}

There are many processes and phenomena in the real world, which are subjected during their development to the short-term external influences. Their duration is negligible compared with the total duration of the studied phenomena and processes. Therefore, it can be assumed that these external effects are "instantaneous", i.e. they are in the form of impulses. The investigation of such "leaps and bounds" developing dynamical states is a subject of different sciences: mechanics, control theory, pharmacokinetics, epidemiology, population dynamics, economics, ecology, etc., [1-5].

\section{There Are Many Examples}

- Operation of a damper, subjected to the percussive effects.

- Change of the valve shutter speed in its transition from open to closed state. effects.

Fluctuations of pendulum system in the case of external impulsive

- Percussive model of a clock mechanism.

- Percussive systems with vibrations.

- Relaxational oscillations of the electromechanical systems.

- Electronic schemes.

- Remittent oscillator, subjected to the impulsive effects.

- Dynamic of a system with automatic regulation.

- The passage of the solid body from a given fluid density to another fluid density.

- Control of the satellite orbit, using the radial acceleration.

- Change of the speed of a chemical reaction in the addition or removal of a catalyst.

- Disturbances in cellular neural networks.

- Impulsive external intervention and optimization problems in the dynamics of isolated populations.

- Death in the populations as a result of impulsive effects.

- Impulsive external interference and the optimization problems in population dynamics of the predator-prey types.

- "Shock" changes of the prices in the closed markets etc.

The use of mathematical apparatus in the form of modeling systems impulsive differential equations in all these cases is natural and binding as a rule.

\section{Brief Scientific Historical Reference}

The mathematical theory of impulsive differential equations develops in two main directions.

First one is introduced in the papers of S. Zavalishchin, A. Sesekin, S.
Drozdenko, A. Halanay, D. Veksler, S. Pandit and S. Deo. The generalized functions (type Dirac delta function) are basic mathematical tools for describing the impulsive effects. The moments of impulsive effect are fixed in advance in these problems, so why this approach is not suitable for modeling systems, where the impulsive moments are determined dynamically, depending on the values of the solutions at every moment of its domain.

Second direction in the research of "leaps" processes was set by Milman $\mathrm{V}$ and Myshkis $\mathrm{A}$, in the sixties of the $20^{\text {th }}$ century with some results for the solutions stability [6]. A number of Ukrainian mathematicians continue the development of this mathematical theory - A. Samoilenko, N. Perestyuk and P. Zabrejk.

Several mathematical schools were created, continuing the scientific research on the fundamental and qualitative theory of impulsive differential equations and their applications in the early eighties and then. We will indicate the groups of: R. Agarval, D. Bainov, P. Eloe, J. Henderson, V. Lakshmikantham, J. Nieto, S. Ntouyas, D. O'Regan, N. Perestyuk, M. Pinto, A. Samoilenko [7-11].

\section{Common Form of Impulsive Systems Differential Equations}

In general, the impulsive equations consist of three parts:

-Differential equation which describes the differentiable part of the solution and usually has the form

$$
\frac{d x}{d t}=f(t, x)
$$

Where the function $f$ is continuous in the domain of the variables $t$ and $x$

condition for consistent determination of the moment of impulsive effect on the solution. In the simplest case, the impulsive moments are fixed in advance. In more complicated and more general case, the impulsive moments are consecutive solutions of the equation

$$
g(t, x(t))=0 \text {, }
$$

Where function is called switching and it is defined and continuous in the extended phase (or only in the phase) space of differential equations, and $x=x(t)$ is a solution of the same.

${ }^{*}$ Corresponding author: Katya Dishlieva, Faculty of Applied Mathematics and Informatics, Technical University of Sofia, Kliment Ohridski 8, Sofia 1000, Bulgaria, E-mail: kgd@tu-sofia.bg

Received July 26, 2012; Accepted July 29, 2012; Published August 02, 2012

Citation: Dishlieva K (2012) Impulsive Differential Equations and Applications. J Applied Computat Mathemat 1:e117. doi:10.4172/2168-9679.1000e117

Copyright: @ 2012 Dishlieva K. This is an open-access article distributed under the terms of the Creative Commons Attribution License, which permits unrestricted use, distribution, and reproduction in any medium, provided the original author and source are credited. 
-The magnitude and direction of the impulsive effect is defined by the impulsive function:

$$
x\left(t_{i}+0\right)=x\left(t_{i}\right)+I\left(x\left(t_{i}\right)\right), i=1,2, \ldots
$$

Function $I$ is called impulsive and it is defined and continuous in the phase space of the considered impulsive system. The impulsive moments $t_{i}, i=1,2, \ldots$, are solutions of the previous equation.

\section{Different Types of Impulsive Differential Equations, Depending on the Way of Determining the Moments of Impulsive Effects}

- Fixed moments of impulsive effects;

- Impulsive moments, which coincide with the moments, at which the integral curve of the equation meets the predefined sets, located in the extended phase space. Frequently these sets are not intersecting hyper surfaces.

- Impulsive moments, which coincide with the moments, at which the trajectory of the equation meets the predefined sets, located in the phase space.

- Impulsive moments, which coincide with the moments, at which the solution minimizes the given function.

- Impulsive moments, which are occasional in their nature and they satisfy a certain law of distribution, etc.

\section{The Specificities, Related to the Study of Impulsive Differential Equations and Difficulties, Arising in Their Investigation:}

- Discontinuity of the solution: There are first-type points of discontinuity, i.e., The leap is limited. Usually, it is assumed that the solution is continuous on the left at the points of impulsive effect.

- Existence of the effect of "beating": In this case, the integral curve or trajectory of the equation meets repeatedly (possibly infinitely many times) impulsive set. Then, it is possible to obtain a specific situation, in which the impulsive moments have a compression point and, therefore, the solution is not continuable to the right from this point. This implies "death" of the solution. Therefore, it is impossible to investigate different aspects of the qualitative theory of these type equations such as: continuous dependence, periodicity, stability, etc in the situation, described above.

- Loss of the autonomous property: Note that, even in the cases, where the right hand sides of the equations with impulses do not depend on time, the impulsive moments are obtained as solutions of equations, involving solution, which (of course) is a function of time. Consequently, these moments depend on the time, including the initial moment. So why, the solution of problem with impulses, which depends on the impulsive moments, is a composite function of the initial point. Thus, the conclusion is that it is not autonomous.

- Fusion of the solutions: usually it takes place after the impulsive effect.

Change of the impulsive moments after changing the initial condition: Different solutions of one and the same impulsive equation (with initial conditions which do not coincide) have different impulsive moments, including the possibility, one of these solutions to be without impulses. It is possible to obtain some differences in the sizes and directions of the basic and perturbed problems after the perturbations in the initial condition.

- Changing the impulsive moments during the interferences of impulsive system: change on the right side, change of the parameters of impulsive system, change of the impulsive functions, etc. The solutions of the basic and corresponding perturbed problem (with the same initial conditions) have different impulsive moments with different in size and direction impulsive effects in the general case.

Accumulation of the errors: the perturbations may have insuperable character and lead to the solutions formation, which differ unlimited from the studied "non perturbed" solution, etc.

\section{Which Problems Should Be Investigated}

In my opinion, the upcoming results on the qualitative theory of differential equations with impulses can be divided into several groups:

1. Transfer of the classical results from the theory of equations without impulsive effects on the equations with impulses. It should be noted that most of these results are obtained. We explicitly emphasize, that the reformulation and proving this type of statements is not always trivial. We explicitly emphasize, that the reformulation and proving this type of statements is not always trivial. This is especially true for the equations with nonfixed moments of impulses.

2. Studying the various phenomena and specific properties of the impulsive equations. Especially, we will point the problems, related to:

- Stability of the solutions after the perturbations on the impulsive moments, impulsive functions, switching functions.

- Periodicity and oscillation properties of the solutions, which are caused by the impulsive effects.

- Solution "death" as a result of impulses.

- Stabilizing and limiting the solutions through the impulsive effects, etc.

3. Modeling and investigation of the properties of dynamic processes, which sharply (intermittently) change its state.

4. Solving optimization problems using these equations. For instance, the problems, which relate the obtaining maximum yield of populations in a limited environment, carrying out chemical reactions with minimal impulsive supply a catalyst, maintaining therapeutic drug concentrations by impulsive changes the concentrations, etc.

Citation: Dishliev A, Dishlieva K, Nenov S (2012) Specific Asymptotic Properties of the Solutions of Impulsive Differential Equations. Methods and Applications, Academic Publications Ltd, 309, ISBN 978-954-2940-09-8.

\section{References}

1. Bainov DD, Dishliev AB, Stamova IM (1996) Lipschitz quasistability of impulsive differential-difference equations with variable impulsive perturbations. J Comput Appl Math 70: 267-277.

2. Dishlieva KG (2012) Continuous dependence of the solutions of impulsive differential equations on the initial conditions and barrier curves. Acta Mathematica Scientia 32: 1035-1052.

3. Dishlieva KG (2011) Differentiability of solutions of impulsive differentia equations with respect to the impulsive perturbations. Nonlinear Analysis: Real World Applications 12: 3541-3551.

4. Lakshmikantham V, Bainov D, Simeonov PS (1989) Theory of Impulsive Differential Equations. World Scientific 6: 288. 
Citation: Dishlieva K (2012) Impulsive Differential Equations and Applications. J Applied Computat Mathemat 1:e117. doi:10.4172/21689679.1000e117

Page 3 of 3

5. Samoilenko A, Perestyuk N (1987) Differential equations with impulsive perturbations.

6. Milman V, Myshkis A (1960) On the stability of motion in the presence of impulses. Siberian Math J 1: 233-237.

7. Agarwal R, Regan OD (2005) A multiplicity result for second order impulsive differential equations via the Leggett Williams fixed point theorem. Appl Math Comput 161: 433-439.

8. enchohra M, Henderson J, Ntouyas S (2006) Impulsive differential equations and inclusions. In: Contemporary Mathematics and its Applications ( $1^{\text {st }}$ edn), Hindawi Publishing Corporations.

9. Eloe P, Henderson J (1997) A boundary value problem for a system of ordinary differential equations with impulse effects. Rocky Mountain J Math 27: 785-799.

10. Naulin R, Pinto M (1997) Quasi-Diagonalization of Linear Impulsive Systems and Applications. J Math Anal Appl 208:281-297.

11. Nieto JJ, Regan OD (2009) Variational approach to impulsive differential equations. Nonlinear Analysis: Real World Applications 10: 680-690. 